\title{
MULTI-SCALE MODELLING OF HETEROGENOUS TEXTILE COMPOSITE STRUCTURES OVER POLYTOPAL TESSELLATED DOMAINS
}

\author{
$\underline{\text { I. Topalidis }}^{1^{*}}$, B. El Said ${ }^{1}$, A. J. Thompson ${ }^{1}$, S. R. Hallett ${ }^{1}$ \\ ${ }^{1}$ Affiliation number one Bristol Composite Institute (ACCIS), Department of Aerospace Engineering, \\ University of Bristol, Queens Building, University Walk, Bristol, BS8 1TR, United Kingdom \\ * ioannis.topalidis@bristol.ac.uk
}

The multi-scale nature of woven composites can be clearly revealed by the strong dependency of the mechanical behaviour on morphological features of lower length scales. Geometrical irregularities in the yarn architecture, induced during the manufacturing stages, alter the mesoscopic material stress field, often dominating the overall material response. To adequately describe major internal geometrical features, common discretisation techniques require to dramatically increase the dimensionality of the problem leading to prohibitive computational demands. On the other hand, the applicability of multi-scale homogenisation techniques, developed to satisfy the need for model order reduction can be limited, due to the large unit cell size of certain weave styles and the loss of periodicity due to local material deformations. To address this, a computationally efficient macroscale modelling approach is proposed, employing a three-dimensional tessellation scheme to obtain a reduced order model that preserves important information about the internal material weave architecture and features.

As an initial step, a kinematic, multi-chain beam model is used to acquire a realistic "as-woven" material internal yarn geometry, from which a surface model of the yarn segments is extracted. The yarn section surfaces feed a spatial tessellation algorithm to generate a set of collectively exhaustive and mutually exclusive polyhedral cells. To exploit the reduced complexity of the tessellated geometry, a mesh of n-faced polyhedral elements [1] is assembled to perform the numerical solution of the

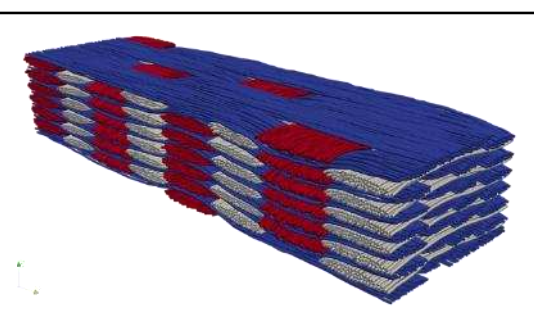

Fig. 1: Material geometry before applying the spatial tessellation. problem domain. The material model follows a multi-scale, bi-material homogenisation approach based on the local meso-structure and the mechanical properties of the two constituents; the fibre and the matrix.

Inaccuracies in predicted results from conventional homogenisation techniques typically arise from smearing out heterogeneities on the yarn level. The capability of the newly proposed framework to overcome such deficiencies in a computationally efficient manner is investigated through benchmark models. Model accuracy, robustness and computational cost are illustrated to evaluate the potential to successfully handle problems at structural component length scales.

\section{Acknowledgements}

The authors would like to acknowledge Rolls-Royce plc for their support of this research through the Composites University Technology Centre at the University of Bristol, UK.

\section{References}

[1] Kumbhar PY, Francis A, Swaminathan N, Annabattula RK, Natarajan S. Development of User Element Routine (UEL) for Cell-Based Smoothed Finite Element Method (CSFEM) in Abaqus. Int J Comput Methods 2020;17:1850128. 